\title{
Modelling of the Formation of Pd-Ni Alloy Nanoparticles by Interdiffusion
}

\author{
A.V. Evteev ${ }^{1, a}$, E.V. Levchenko, ${ }^{1, b}$, I.V. Belova ${ }^{1, c}$ and G.E. Murch ${ }^{1, d}$ \\ ${ }^{1}$ Diffusion in Solids Group, School of Engineering, \\ The University of Newcastle, Callaghan, NSW 2308, Australia \\ aAlexander.Evteev@newcastle.edu.au, ${ }^{\mathrm{b}}$ Elena.Levchenko@newcastle.edu.au, \\ Irina.Belova@newcastle.edu.au, 'Graeme.Murch@newcastle.edu.au
}

\begin{abstract}
Keywords: Molecular dynamics, embedded atom method, interdiffusion, surface segregation,
\end{abstract} structure, palladium, nickel, alloy nanoparticles.

\begin{abstract}
Long-time scale molecular dynamics simulation in combination with the embedded atom method is used to investigate the effect of surface segregation phenomena at $1000 \mathrm{~K}$ on the structure of Pd alloy nanoparticles (of diameter of $\sim 4.5 \mathrm{~nm}$ ) containing $~ 30$ at. $\% \mathrm{Ni}$. A core-shell f.c.c. structure was chosen as the initial state wherein a core of $\mathrm{Ni}$ atoms is surrounded by shell of $\mathrm{Pd}$ atoms. It is found that such nanoparticles form a surface-sandwich structure by interdiffusion. In this structure, the Ni atoms, which mostly accumulate in a layer just below the surface and at the same time are located in the centres of interpenetrating icosahedra to generate a subsurface shell as a Kagomé net. Meanwhile, the Pd atoms occupy the vertices of the icosahedra and cover this Ni layer from inside and outside as well as being located in the core of the nanoparticle forming (according to alloy composition) a Pd-rich solid solution with the remaining $\mathrm{Ni}$ atoms. The total atomic fraction involved in building up the shell of the nanoparticle in the form of the Ni Kagomé net layer covered on both side by Pd atoms can be estimated at $70 \%$.
\end{abstract}

\section{Introduction}

Bimetallic Pd-Ni nanoparticles are currently attracting a great deal of interest due to their physical and chemical properties [1-5], which are determined by their size, shape, structure and composition. In particular, it is well-known that $\mathrm{Pd}$ is a metal with important existing and potential applications as a catalyst in heterogeneous catalysis. The use of $\mathrm{Pd}$ as a single active metal component in catalysis has received considerably attention on the basis of its remarkable activity for oxidation reactions and the availability of cleaner fuels, but at the same time it has negative economical aspects due to its high cost $[4,6]$. An interesting approach in these respects consist of alloying Pd with lower cost and higher surface energy metals [6], since it would be economically attractive to design bimetallic catalyst nanoparticles in which the precious and catalytic Pd atoms segregate to the surface. Promising results in this sense have been obtained by using Ni [2-4, 7-10].

The surface segregation phenomena at low indices surfaces in different Pd-Ni crystal systems have been studied by several experimental techniques [7-13] and by modelling with different theoretical approaches [7, 14-21]. Most of the measurements and calculation showed a considerable segregation of Pd to the surfaces and oscillations in the atomic layer composition near the surfaces with the second layer enriched by $\mathrm{Ni}$ atoms.

It is clear in case of alloy nanoparticles the surface segregation is one of the central concepts when structure and properties are to be described and understood. Furthermore, as was noted in [21], in materials with a large fraction of the atoms at surfaces the thermodynamic driving force towards surface segregation might be large. This may lead to excessive solid-state diffusion and hence possibly to an increased density of faults and defects in the crystal structure, moreover, bulk order may be broken at the grain boundaries and interfaces. Understanding and controlling of the structure of alloy nanoparticles are important in both fundamental science and technological applications. Atomistic simulation techniques such as molecular dynamics (MD) have become a powerful tool in the field of nanotechnology by providing physical insight in understanding phenomena on an atomic scale and predicting many of the properties of nanomaterials.

In the present study, long-time scale molecular dynamics simulation in combination with the embedded atom method (EAM) is used to investigate the effect of surface segregation phenomena on the structure of Pd alloy nanoparticles (of diameter of $\sim 4.5 \mathrm{~nm}$ ) containing $\sim 30$ at. $\% \mathrm{Ni}$. 


\section{The Model}

The EAM represents the total energy of an atomic system in the form [22]

$$
E_{\text {tot }}=\sum_{i}\left[F_{i}\left(\bar{\rho}_{i}\right)+\frac{1}{2} \sum_{j \neq i)} \phi_{i j}\left(r_{i j}\right)\right] \text {. }
$$

In this expression, $\bar{\rho}_{i}$ is the host electron density at atom $i$ due to the remaining atoms of the system, $F_{i}\left(\bar{\rho}_{i}\right)$ is the energy to embed atom $i$ into the background electron density $\bar{\rho}_{i}$ and $\phi_{i j}\left(r_{i j}\right)$ is a pair-interaction representing the core-core repulsion between atoms $i$ and $j$ separated by the distance $r_{i j}$. The electron density is approximated by the superposition of atomic densities,

$$
\bar{\rho}_{i}=\sum_{j(\neq i)} \rho_{j}\left(r_{i j}\right),
$$

where $\rho_{j}\left(r_{i j}\right)$ is the atomic electron density of atom $j$ at the distance $r_{i j}$ from the nucleus. The embedding function $F_{i}\left(\bar{\rho}_{i}\right)$ is universal in that it does not depend on the source of the background electron density. Thus the same embedding function is used to calculate the energy of an atom in an alloy that is used in the pure material. The form of the pair-repulsion term has been studied from first principles by Daw [23]. This analysis shows that the pair-interaction between two different species can be approximated by the geometric mean of the pair-interaction for the individual species. This observation, along with the Coulombic origin of the pairinteraction term, suggest writing the pair-interaction between atoms in terms of effective charges $Z_{i}\left(r_{i j}\right)$ as:

$$
\phi_{i j}\left(r_{i j}\right)=Z_{i}\left(r_{i j}\right) Z_{j}\left(r_{i j}\right) / r_{i j} \text {. }
$$

To apply this method, the embedding functions, pair-repulsions (or effective charges) and atomic electron densities must be known. The details of the parameterization used in our calculations of the particular sets of EAM functions developed by Wilson and Bisson (EAM-WB) by fitting to bulk equilibrium solid properties (lattice constant, heat of sublimation, elastic constants, vacancy formation energy, bcc-fcc energy difference and the hydrogen heat of solution) of $\mathrm{Ni}$ and $\mathrm{Pd}$ are presented in the Appendix of [24]. Furthermore, these sets of EAM functions provided a good description of the liquid state of $\mathrm{Ni}$ and $\mathrm{Pd}$ [24], and so must include reasonable values of the anharmonicity of the interatomic interactions.

Some very helpful qualitative predictions of the surface segregation behaviour in alloys are possible from the macroscopic thermodynamic point of view. In randomly ordered bimetallic alloys surface segregation phenomena must be driven by the differences in surface energy for various surface compositions. Therefore, the surface energies of the low indices faces (100), (110) and (111) of the two pure metals have been calculated using the EAM-WB to verify that the method gives reasonable values. The surface energy is computed by minimizing the energy of a slab of atoms with respect to all atomic positions to incorporate surface relaxation effects. The surface energy is then difference between the energy of the slab and that of an equal number of atoms in the bulk. The resulting values of the surface energies of $\mathrm{Ni}$ and $\mathrm{Pd}$ are presented in Table 1. For both metals, the close-packed (111) face has the lowest energy, followed by the (100) and (110) faces. This Table also contains the estimates [25] of the average solid metal surface energy based on liquid metal surface energies experimental data (see for example, [26]). The experimental values have been crudely extrapolated from the melt temperature to $0 \mathrm{~K}$ [25]. This agreement between theory and experiment is reasonable since there is the presence of less energetically favourable faces and defects on the average surface for which these estimates are intended. The theoretical values are for ideal low indices faces which one would expect to have a below average surface energy. More important for segregation phenomena, the theory predicts the correct relative value of the surface energies for the two metals [27].

Table 1. Surface energies of the low indices faces of Ni and Pd predicted by EAM-WB potentials in comparison with experimental data [25] and [26] in units of $\mathrm{J} / \mathrm{m}^{2}$.

\begin{tabular}{|c|c|c|c|c|c|c|}
\hline & \multicolumn{3}{|c|}{$\mathrm{Ni}$} & \multicolumn{3}{c|}{$\mathrm{Pd}$} \\
\cline { 2 - 7 } & $(111)$ & $(100)$ & $(110)$ & $(111)$ & $(100)$ & $(110)$ \\
\hline EAM-WB & 1.30 & 1.54 & 1.70 & 1.06 & 1.25 & 1.34 \\
\hline Experiment & \multicolumn{3}{|c|}{$2.37[25], 1.77[26]$} & \multicolumn{3}{c|}{$2.00[25], 1.48[26]$} \\
\hline
\end{tabular}


The heats of solution and the deviations of the lattice constant from Vegard's law for bulk compositions of $\mathrm{Pd}-\mathrm{Ni}$ alloys have also been determined and compared with experimental data $[28,29]$. The results of calculations for the heat of solution (i.e. the difference between the enthalpy of the alloy and the linear interpolation of the enthalpy of the pure metals) are given as the solid line in Fig. 1(a). For comparison the experimental values [28] are presented as open circles. The agreement between these two is quite good; the deviation from each other at any composition does not exceed $0.025 \mathrm{eV} /$ at. Furthermore, in all cases, the heat of solution is rather small. The small heat of solution is quite plausible, since $\mathrm{Pd}$ and $\mathrm{Ni}$ are neighbours in the periodic system and exhibit a continuous mutual solid solubility without atomic ordering. Fig. 1(b) shows the deviations of the lattice constant from Vegard's law. Again the EAM-WB results (solid line) and experimental values [29] (open circles) are in good agreement. It is seen from Fig. 1(b)

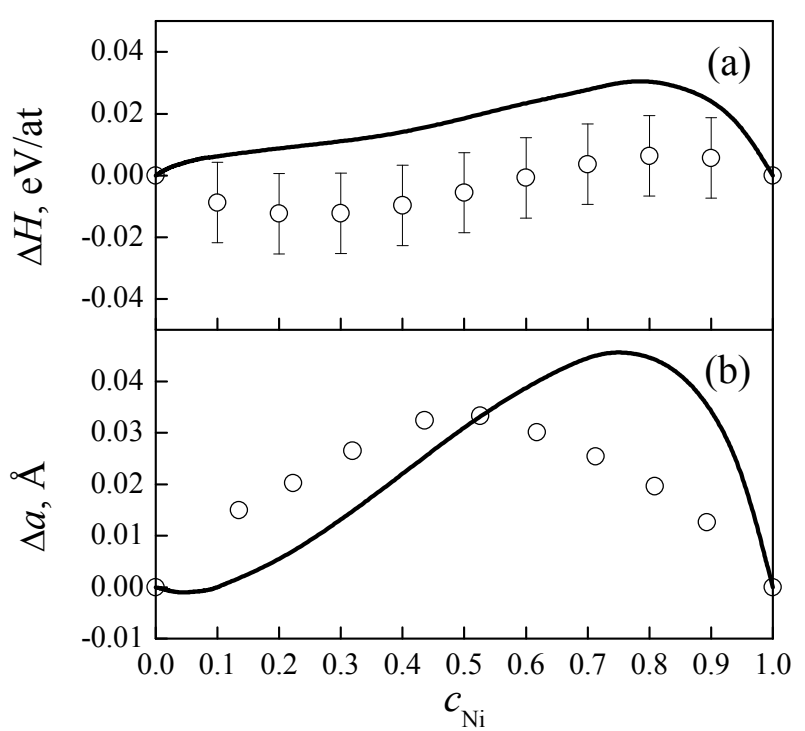

Fig. 1. Heat of solution $\Delta H$ (a) and deviation of the lattice constant from Vegard's law $\Delta a$ (b) for bulk Pd-Ni alloys as a function of composition $c_{\mathrm{Ni}}$. Solid line: results using the EAM-WB; open circles: experimental data [28] (a) and [29] (b). the lattice constants are correctly predicted by EAM-WB potentials to within $<0.015 \AA$ and $<0.025 \AA$ for Pd-rich and Ni-rich alloys, respectively. Thus an application of the EAM-WB to the calculation on concentrated substitutional Pd-Ni alloys, starting from results for the pure components, appears to be reasonable. In this, the EAM-WB is much better than the EAM functions determined in the earlier work of Daw and Baskes [22] for pure Ni and Pd. Unfortunately, the last EAM functions yield poor agreement between theory and experiment for the calculation on concentrated substitutional Pd-Ni alloys [30].

A spherical core-shell f.c.c. structure was chosen as the initial state for bimetallic $\mathrm{Pd}(\approx 66 \%)-\mathrm{Ni}(\approx 34 \%)$ nanoparticle wherein a core with radius of $\sim 1.4 \mathrm{~nm}$ of $1088 \mathrm{Ni}$ atoms is surrounded by a shell with external radius of $\sim 2.2 \mathrm{~nm}$ of $2132 \mathrm{Pd}$ atoms. Then the static relaxation procedure was applied to accommodate the core and shell atoms especially at the interface, since Pd atoms (the equilibrium bulk lattice constant is $3.89 \AA$ ) are noticeably larger than Ni atoms (the equilibrium bulk lattice constant is $3.52 \AA$ ) with difference in atomic sizes of $\sim 10 \%$. Following this, atoms were given initial velocities according to the Maxwell distribution at the temperature of $1000 \mathrm{~K}$ and the MD procedure of isothermal annealing was performed. The MD procedure consisted of a numerical integration of the equations of atomic motion according to the Verlet algorithm [31] with a time step $\Delta t=1.5 \mathrm{fs}$. During the simulation momentum and angular momentum of the whole system were conserved at zero values. Periodically, without an effect on the continuity of the annealing procedure, the system was transferred to a state at $T=0 \mathrm{~K}$ where atoms occupied equilibrium positions in local potential minima by making use of the static relaxation method. After this, the atomic movements and structure transformations occurring in the model were analyzed.

\section{Results and Discussion}

Just after the static relaxation of the initial Pd-Ni nanoparticle model, the position of the interface between the core and shell can be approximated by the spherical layer with radius $14.5 \AA$. Actually, because of large lattice constant mismatches, the interface here is strongly distorted with quite a wide range of distances with the middle at radius $\sim 14.5 \AA$. During of the interdiffusion process, the distorted crystal order of the nanoparticle starts to be broken at the interface rather quickly, and then the structure of the whole nanoparticle evolves to a disordered state. The interdiffusion process with formation of a disordered Pd-rich solid solution has almost finished after the first $30 \mathrm{~ns}$ of MD annealing at $1000 \mathrm{~K}$, as can be seen in Fig. 2(a). However, the potential energy $U_{0}$ of the model after static relaxation (see Fig. 2(b)) proceeds to decrease continuously. This is evidence of further structure transformations occurring in the 
model. Monitoring of the structure of the nanoparticle by the Voronoi polyhedron analysis shows a significant increasing of the icosahedral short-range order in the model during annealing (Fig. 3(a)). The centres of the icosahedral cages are almost always the smaller Ni atoms. The most favourable sites for $\mathrm{Ni}$ icosahedra are placed in a subsurface shell. Indeed, after $\sim 0.5 \mu \mathrm{s}$ of the annealing, $\sim 71 \%$ of all $\mathrm{Ni}$ atoms are located in the centres of the perfect icosahedra. Among the $790 \mathrm{Ni}$ atoms gone from initial core to the shell the icosahedra fraction is $\sim 78 \%$ while it is $\sim 52 \%$ of the $298 \mathrm{Ni}$ atoms remaining in the core. In total, in the time $\sim 0.5 \mu \mathrm{s}, \sim 90 \%$ of all atoms in the system are involved in the construction of the icosahedra with a Ni atom in the centre (see Fig. 3 (b)).

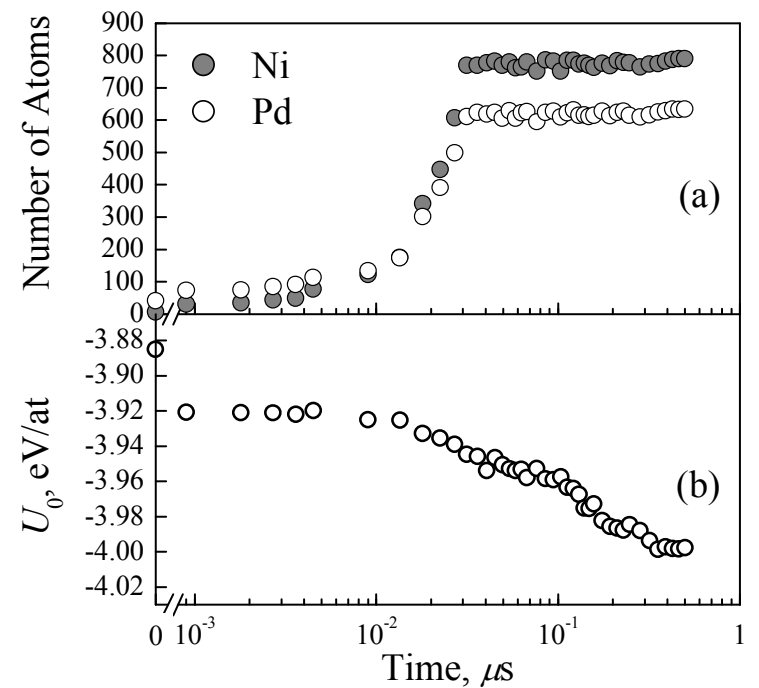

Fig. 2. Time dependences of (a) the number of $\mathrm{Ni}$ atoms gone from core to shell as well as $\mathrm{Pd}$ atoms gone from shell to core across the spherical layer with radius $14.5 \AA$ (accepted as position of the initial interface) and (b) potential energy of the model after static relaxation.

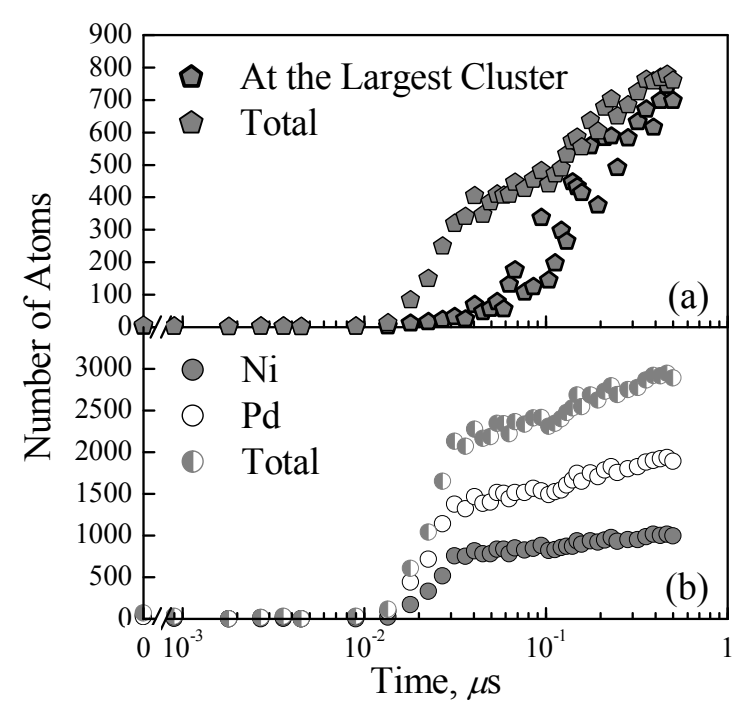

Fig. 3. Time dependences of the number of (a) $\mathrm{Ni}$ atoms having an icosahedron as a coordination polyhedron in total as well as constituting the largest cluster from interpenetrating icosahedra and (b) number of atoms involving in the construction of the icosahedra with a $\mathrm{Ni}$ atom in the centre.

It was established that $\mathrm{Ni}$ atoms incoming by interdiffusion to the shell begin to accumulate in a layer just below the surface, and then locate in the centres of interpenetrating icosahedra in order to generate a subsurface layer (shell) as a Kagomé net (Fig. 4(a)), which is almost completely covered by Pd atoms on both sides. We recall that planar layers of Kagomé nets are the basic structure of the well-known bulk Frank-Kasper phases of certain intermetallic alloys [32]. It is clear that according to the Euler theorem it is impossible to obtain a perfect closed shell from a Kagomé net; therefore, it will always have the 'sequence faults'. In fact, the forming of the Kagomé net shell in subsurface of the nanoparticle is realised by nucleation, growth and coalescence of $2 \mathrm{D}$ islands (clusters) of the interpenetrating icosahedra. This subsurface Kagomé net shell of $\mathrm{Ni}$ atoms efficiently allows the minimization of the nanoparticle surface energy by increasing of the average number of nearest neighbours of Pd surface atoms in comparison with the close-packed f.c.c. (111) surface. Indeed, the Ni Kagomé net layer $(K)$ is covered from each side by two Pd layers ( $A^{\prime}$ and $A^{\prime \prime}$ ), which can be obtained by splitting of a layer $A$ - close packed f.c.c. (111) layer (see Fig. 4(b)). The forming of such a five-layer $A^{\prime} A^{\prime \prime} K A^{\prime \prime} A^{\prime}$ surface structure results in a third of surface $\mathrm{Pd}$ atoms having increased their number of nearest neighbours from 9 (the close packed f.c.c. (111) surface) to 13 (see also Table 2). The number of Pd atoms in each couple layers $A^{\prime}$ and $A^{\prime \prime}$ coincides with the number of $\mathrm{Ni}$ atoms in the Kagomé net layer, therefore, the total atomic fraction involving in build up of the five-layer $A^{\prime} A^{\prime \prime} K A^{\prime \prime} A^{\prime}$ surface structure in the nanoparticle with consideration of some imperfection of icosahedral order in Kagomé net layer can be estimated at $\sim 70 \%$. Thus, only $~ 30 \%$ atoms are located in the core of nanoparticle and form there, according to alloy composition, a non-crystalline Pd-rich solid solution with quite strongly developed icosahedral short-range order. This final arrangement of the nanoparticle is the result of competition between the shell ( $70 \%$ atoms $)$ and the core $(\sim 30 \%$ atoms $)$ contributions to excess energy. 


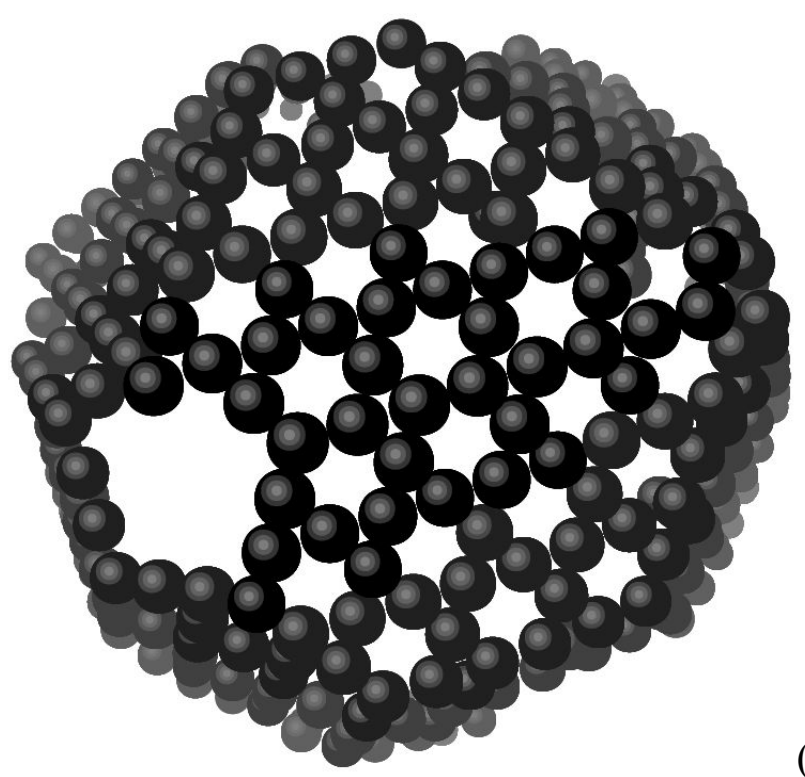

(a)

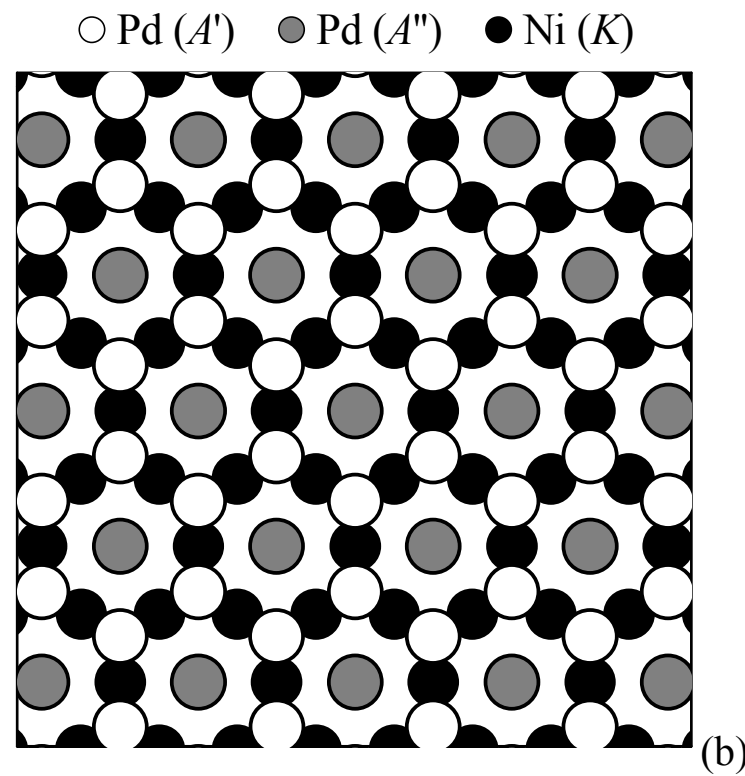

Fig. 4. (a) - Snapshot of the Ni subsurface shell having a structure of the Kagomé net with 'sequence faults' after $\sim 0.3 \mu \mathrm{s}$ of annealing at $T=1000 \mathrm{~K}$. The great majority of Ni atoms of the subsurface shell are located in the centres of interpenetrating icosahedra and have $4 \mathrm{Ni}$ and $8 \mathrm{Pd}$ nearest neighbours. For better clarity only a half of the shell and only $\mathrm{Ni}$ atoms at centres of icosahedra are shown on the perspective projection with size and grey-scale graduation. (b) - Illustration of atomic positions of $\mathrm{Pd}$ and $\mathrm{Ni}$ atoms on a perfect fragment of surface structure $A^{\prime} A^{\prime \prime} K A^{\prime \prime} A^{\prime}$. Layers $A^{\prime}$ and $A^{\prime \prime}$ of $\mathrm{Pd}$ can be obtained by splitting of a layer $A$ - close packed f.c.c. (111) layer; layer $K$ of $\mathrm{Ni}$ is a Kagomé net layer.

In conclusion, we note because it may become energetically unfavourable with increase of the size of the nanoparticle or deviation of the ratio of $\mathrm{Pd}$ and $\mathrm{Ni}$ atoms from 2:1 the core contribution to the excess energy overcomes the shell contribution. Thus the Pd-Ni alloy nanoparticle structure is likely to depend strongly on alloy composition and nanoparticle size. However, it would be useful to obtain experimentally such Pd-Ni nanoparticles and nanowires, which should present unusual physical and chemical properties. Furthermore, of interest for applications would be success in experimentally creating a five-layer $A^{\prime} A^{\prime \prime} K A^{\prime \prime} A^{\prime} \mathrm{Pd}_{2} \mathrm{Ni}$ nanofilm from which a five-layer $A^{\prime} A^{\prime \prime} K A^{\prime \prime} A^{\prime} \quad \mathrm{Pd}_{2} \mathrm{Ni}$ hollow nanosphere and nanotube might be synthesized. The main structure parameters of the hypothetical five-

Table 2. The main structure parameters of the five-layer $A^{\prime} A^{\prime \prime} K A^{\prime \prime} A^{\prime} \mathrm{Pd}_{2} \mathrm{Ni}$ nanofilm model.

\begin{tabular}{|c|c|c|c|c|c|c|c|}
\hline \multirow{3}{*}{ 造 } & \multirow{3}{*}{ 竓 } & \multirow{3}{*}{$\begin{array}{l}\text { Atomic } \\
\text { Fraction }\end{array}$} & \multicolumn{5}{|c|}{ Nearest Neighbours } \\
\hline & & & \multirow{2}{*}{$r, \AA$} & \multicolumn{2}{|c|}{$\mathrm{Pd}$} & $\mathrm{Ni}$ & \multirow{2}{*}{ Total } \\
\hline & & & & $A^{\prime}$ & $A^{\prime \prime}$ & $K$ & \\
\hline \multirow{6}{*}{$\mathrm{Pd}$} & \multirow{3}{*}{$A^{\prime}$} & & 2.51 & - & - & 3 & \multirow{3}{*}{9} \\
\hline & & & 2.73 & 3 & - & - & \\
\hline & & & 2.85 & - & 3 & - & \\
\hline & \multirow{3}{*}{$A^{\prime \prime}$} & & 2.62 & - & 1 & - & \multirow{3}{*}{13} \\
\hline & & & 2.71 & - & - & 6 & \\
\hline & & & 2.85 & 6 & - & - & \\
\hline \multirow{3}{*}{$\mathrm{Ni}$} & \multirow{3}{*}{$K$} & \multirow{3}{*}{3} & 2.37 & - & - & 4 & \multirow{3}{*}{12} \\
\hline & & & 2.51 & 4 & - & - & \\
\hline & & & 2.71 & - & 4 & - & \\
\hline
\end{tabular}
layer $A^{\prime} A^{\prime \prime} K A^{\prime \prime} A^{\prime} \mathrm{Pd}_{2} \mathrm{Ni}$ nanofilm obtained by static relaxation method with EAM-WB potentials are listed in Table 2 and they are very close to ones of the five-layer $A^{\prime} A^{\prime \prime} K A^{\prime \prime} A^{\prime}$ shell structure obtained for investigating in this work Pd-Ni nanoparticle model. Finally, we can suppose that surface reconstruction by surface segregation forming a five-layer $A^{\prime} A^{\prime \prime} K A^{\prime \prime} A^{\prime}$ surface structure may occur in (111) oriented $(A B C A B C \ldots) \mathrm{Pd}_{100-x} \mathrm{Ni}_{x}$ crystal alloy with a composition close to $x \approx 33 \%: \underbrace{A^{\prime} A_{\mathrm{Ni}}^{\prime \prime}}_{\mathrm{Pd}} \underbrace{A^{\prime \prime}}_{\mathrm{Pd}} \underbrace{B C A B C A \ldots}_{\mathrm{Pd}_{100-x} \mathrm{Ni}_{x}(x \approx 33 \%)}$.

\section{Summary}

By long-time scale molecular dynamics simulation in combination with the embedded atom method we have demonstrated that a novel kind of the structure of $\operatorname{Pd}_{100-x} \mathrm{Ni}_{x}(x \sim 30 \%)$ alloy nanoparticles of diameter of $\sim 4.5 \mathrm{~nm}$ can be obtained by interdiffusion from an initial state with the f.c.c. structure wherein a core of $\mathrm{Ni}$ atoms is surrounded by shell of Pd atoms. In this structure, the Ni atoms, which mostly accumulate in a layer just below the surface, at the same time, are located in the centres of interpenetrating 
icosahedra to generate a subsurface layer, $K$, as a Kagomé net. Meanwhile, the Pd atoms occupy the vertices of the icosahedra and cover this Ni layer from inside and outside by two Pd layers $A^{\prime}$ and $A^{\prime \prime}$, which can be obtained by splitting of a layer $A$ - close packed f.c.c. (111) layer as well as being located in the core of the nanoparticle forming (according to the alloy composition) a Pd-rich solid solution with the rest of the $\mathrm{Ni}$ atoms. The forming of the five-layer $A^{\prime} A^{\prime \prime} K A^{\prime \prime} A^{\prime}$ surface structure allows the efficient minimization of the nanoparticle surface energy. The total atomic fraction involved in the building up of the five-layer shell of the nanoparticle can be estimated at $\sim 70 \%$. Thus, our results indicate a new possibility for controlling the chemical ordering in Pd-Ni structures at the nanoscale level. For example, the synthesis of a $\mathrm{Pd}_{2} \mathrm{Ni}$ hollow nanosphere and nanotube might be facilitated from a five-layer $A^{\prime} A^{\prime \prime} K A^{\prime \prime} A^{\prime} \mathrm{Pd}_{2} \mathrm{Ni}$ nanofilm.

\section{Acknowledgements}

This research was supported by the Australian Research Council. One of us (E.V.L.) wishes to thank the University of Newcastle for the award of a Research Fellowship.

\section{References}

[1] N. Nunomura, H. Hori, T. Teranishi, M. Miyake, S. Yamada: Phys. Lett. A Vol. 249 (1998), p. 524.

[2] S. Takenaka, Y. Shigeta, E. Tanabe, K. Otsuka: J. Phys. Chem. B Vol.108 (2004), p. 7656.

[3] S. Sao-Joao, S. Giorgio, J.M. Penisson, C. Chapon, S. Bourgeois, C Henry: J. Phys. Chem. B Vol.109 (2005), p. 342.

[4] A.B. Hungría, J.J. Calvino, J.A. Anderson, A. Martínez-Arias: Appl. Catal. B Vol. 62 (2006), p. 359.

[5] R. Jianwei, L. Shijun. L. Junmin: Chinese Science Bulletin Vol. 51 (2006), p. 2959.

[6] A. Martínez-Arias, J.C. Coneza, M. Fernández-García, J.A. Anderson, in: Supported Metals in Catalysis, edited by J.A. Anderson and M. Fernández-García, Imperial College Press (2005).

[7] P. Miegge, J.L. Rousset, B. Tardy, J. Massardier, J.C. Bertolini: J. Catal. Vol. 149 (1994), p. 404.

[8] P. Herman, J.M. Guigner, B. Tardy, Y.Jugnet, D. Simon, J.C. Bertolini: J. Catal. Vol. 163 (1996), p. 169.

[9] A.C. Michel, L. Lianos, J.L. Rousset, P. Delichère, N.S. Prakash, J. Massardier, Y. Jugnet, J.C. Bertolini: Surf. Sci. Vol. 416 (1998), p. 288.

[10] L. Porte, M. Phaner-Goutorbe, J.M. Guigner, J.C. Bertolini: Surf. Sci. Vol. 424 (1999), p. 262.

[11] G.N. Derry, C.B. McVey, P.J. Rous: Surf. Sci. Vol. 326 (1995), p. 59.

[12] M. Abel, Y. Robach, J.C. Bertolini, L. Porte: Surf. Sci. Vol. 454-456 (2000), p. 1.

[13] G.N. Derry, R. Wan: Surf. Sci. Vol. 566 (2004), p. 862.

[14] J.L. Rousset, J.C. Bertolini, P. Miegge: Phys. Rev. B Vol. 53 (1996), p. 4947.

[15] B.C. Khanra, J.C. Bertolini, J.L. Rousset: J. Mol. Catal. A Vol. 129 (1998), p.233.

[16] A. Christensen, A.V. Ruban, H.L. Skriver: Surf. Sci. Vol. 383 (1997), p. 235.

[17] L.V. Poyurovskii, A.V. Ruban, I.A. Abrikosov, Y.K. Vekilov, B. Johansson: JEPT Lett. Vol. 73 (2001), p.415.

[18] J.-S. Filhol, D. Simon, P.Sautet: Phys. Rev. B Vol. 64 (2001), p. 85412.

[19] S. Helfensteyn, J. Luyten, L. Feyaerts, C. Creemers: Appl. Surf. Sci. Vol. 212 (2003), p. 844.

[20] A.A. Dmitriev, A.V. Evteev, V.M. Ievlev, A.T. Kosilov: Doklady Physics Vol. 49 (2004), p. 292.

[21] O.M. Løvvik: Surf. Sci. Vol. 583 (2005), p. 100.

[22] M.S. Daw and M.I. Baskes: Phys. Rev. B Vol. 29 (1984), p. 6443.

[23] M.S. Daw: Phys. Rev. B Vol. 39 (1989), p. 7441.

[24] S.M. Foiles: Phys. Rev. B Vol. 32 (1985), p. 3409.

[25] W.R. Tyson, W.A. Miller: Surf. Sci. Vol. 62 (1977), p. 267.

[26] A. Zangwill: Physics at Surfaces (Cambridge University Press, Cambridge 1988).

[27] S.M. Foiles: Phys. Rev. B Vol. 32 (1985), p. 7685.

[28] R. Hultgren, P.D. Desai, D.T. Hawkins, M. Gleiser, K.K. Kelly: Selected Values of the Thermodynamic Properties of Binary Alloys (American Society for Metals, Metals Park, Ohio 1973).

[29] L.R. Bidwell, R. Speiser: Acta Cryst. Vol. 17 (1964), p. 1473.

[30] P.R. Maarleveld, P.B. Kaars, A.W. Weeber, H. Bakker: Physica B Vol. 142 (1986), p.328.

[31] L. Verlet: Phys. Rev. Vol. 159 (1967), p. 98.

[32] F.C. Frank and J.S. Kasper: Acta Cryst. Vol. 11 (1958), p. 184; Vol. 12 (1959), p. 483. 